\title{
Apports de la simulation numérique tridimensionnelle dans les études de tunnels
}

\author{
Jean-Pierre Janin ${ }^{\star}$ \\ Terrasol, immeuble le Crystallin, 191/193 cours Lafayette, 69458 Lyon cedex 06, France
}

\begin{abstract}
Résumé - L'excavation d'ouvrages souterrains et, en particulier, de tunnels représente un problème tridimensionnel complexe, dans lequel la réaction du massif dépend de différents facteurs, comme la nature et les caractéristiques du sol, la géométrie de l'ouvrage à réaliser et la méthode de creusement adoptée. Dans un contexte urbanisé, la réalisation de ces ouvrages pose le problème de l'interaction avec les bâtiments en surface et les structures déjà existantes en souterrain. La pratique courante de dimensionnement des tunnels, notamment vis-à-vis des déformations, s'appuie principalement sur l'approche numérique en déformations planes, basée sur les principes de la méthode convergence-confinement. Cependant, cette méthode, dont la fiabilité est directement liée au choix du taux de déconfinement, est souvent utilisée dans des situations où les hypothèses de base ne sont pas respectées. Cet article présente ainsi quelques exemples qui montrent comment la modélisation 3D est devenue, aujourd'hui, un outil indispensable, aux bureaux d'étude géotechnique, pour étudier certains problèmes dans toute leur complexité et se rapprocher au mieux de la réalité. Les études présentées traitent des ouvrages réalisés en méthode traditionnelle et mécanisée, où la problématique des prévisions des tassements et des interactions avec les structures existantes est un enjeu prioritaire du projet.
\end{abstract}

Mots clés : tunnels / simulations numériques 3D / prévision des tassements / interaction sol-structure

\begin{abstract}
Contributions of the three-dimensional numerical simulation in tunnels studies. The excavation of underground structures and, in particular, tunnels is a complex three-dimensional problem, wherein the ground reaction depends on various factors such as the nature and characteristics of the soil, the geometry of the work and the excavation method adopted. In an urban context, the construction of these works poses the problem of interaction with surface buildings and existing underground structures. The current practice of tunnels projects design, particularly concerning ground deformations, is mainly based on the twodimensional analysis, in a cross-section with in-plane deformations combined with the convergenceconfinement method. However, this method, wherein the validity of the results is based on the correct choice of the stress release coefficient $\lambda$, is often used in situations where the basic assumptions are not respected. This article provides some examples showing how 3D modeling has become an indispensable tool today, for geotechnical design engineers, to study certain issues in all their complexity and to better approximate the reality. Studies presented deal with works carried out in traditional and mechanized method, where the prediction of settlements and interactions with existing structures is a priority project issue.
\end{abstract}

Keywords: tunnels / 3D numerical simulations / settlement predictions / soil-structure interaction

\section{Introduction}

Alors que les agglomérations urbaines se densifient de plus en plus, la réalisation d'ouvrages souterrains, tels que parkings, tunnels métropolitains, routiers, ferroviaires, etc., est devenue un enjeu prioritaire dans le cadre des politiques de réorganisation des espaces, d'amélioration de la viabilité et du développement des transports en commun. Le creusement de ces ouvrages pose la question de l'interaction avec les bâtiments en surface

\footnotetext{
$\bar{\star}$ Auteur de correspondance : jp.janin@terrasol.com
}

et les structures déjà existantes en souterrain. L'approche de l'ingénieur doit ainsi tenir compte de deux problématiques: d'une part, assurer le bon déroulement des travaux en préservant la stabilité de l'excavation et, d'autre part, contrôler les déformations du massif encaissant pour limiter l'interaction avec les constructions avoisinantes. Ainsi, dès la phase de projet, une estimation correcte des mouvements engendrés dans le massif et des déformations induites dans les structures existantes est essentielle afin de bien concevoir la méthodologie de réalisation de l'ouvrage. Celle-ci pourra ensuite être adaptée sur la base des mesures prises in situ pendant les travaux, selon les principes de la méthode observationnelle. 
Les mouvements engendrés par le creusement d'un tunnel sont la traduction de phénomènes complexes dépendant de la nature du sol, des méthodes de creusement et de la géométrie de l'ouvrage à réaliser. Cette problématique peut être abordée par des approches empiriques, analytiques et numériques. Dans le cadre des approches empiriques, les observations menées in situ montrent que la cuvette transversale de tassement en surface peut être généralement approximée par la courbe de Gauss (Schmidt, 1969; Peck, 1969; Attewell, 1977; Clough et Schmidt, 1981; O’Reilly et New, 1982; Rankin, 1988), pouvant être entièrement décrite par la donnée de deux paramètres, le point d'inflexion $i$ et le rapport entre le volume de la cuvette générée en surface et le volume excavé $\left(V_{\text {Loss }}\right)$. Cependant, ces deux paramètres varient significativement en fonction de plusieurs facteurs, tels que la stratigraphie du massif, la méthode de creusement, la forme de la galerie, etc. Les méthodes analytiques, de leur côté, sont des outils d'analyse utiles mais ils présentent des limitations d'application à cause des hypothèses restrictives sur lesquelles les formulations se basent. Dans ce cadre, la méthode convergence-confinement (Panet, 1995), largement utilisée dans la pratique courante de dimensionnement des tunnels, se distingue par le fait de prendre en compte l'aspect tridimensionnel du problème du creusement d'un tunnel par une simplification basée sur le concept du taux de déconfinement $\lambda$. Toutefois, cette méthode, implémentée dans les calculs numériques bidimensionnels, est trop souvent utilisée dans des situations de tunnel peu profond, de massif hétérogène et anisotrope, ou d'excavation phasée..., où les hypothèses de base ne sont pas respectées. Le recours à une modélisation tridimensionnelle devient alors incontournable pour étudier le problème dans toute sa complexité et se rapprocher au mieux de la réalité. Ainsi, l'hétérogénéité du massif, la réelle géométrie de l'ouvrage, le phasage des travaux, l'interaction avec les structures existantes et l'ensemble des phénomènes mis en jeu peuvent être considérés.

Dans le cadre d'une thèse « CIFRE » (Janin, 2012), un suivi régulier du chantier du deuxième tube de Toulon a été réalisé. Les études en rétro-analyse numériques, sur les mesures enregistrées in situ, ont mis ensuite en évidence les atouts de l'approche tridimensionnelle, permettant de bien évaluer le déconfinement et les déformations engendrées dans le massif. Les résultats de ce travail ont été ensuite appliqués à plusieurs projets sensibles où le recours à un modèle $3 \mathrm{D}$ a été la clé de la réussite des études.

Cet article n'a pas la prétention d'illustrer des nouvelles méthodologies de calcul mais il vise à montrer comment aujourd'hui, grâce au développement des ordinateurs et des codes de calcul, les approches 3D, qui relevaient encore il y a peu de temps du domaine de la recherche universitaire, ont pu être étendues au domaine de la pratique professionnelle en bureaux d'étude.

\section{Tunnel urbain en méthode traditionnelle: deuxième tube de Toulon}

Le tube sud du tunnel de Toulon, qui relie les autoroutes A50 et A57, a été excavé en milieu fortement urbanisé, sous faible couverture (de 15 à $40 \mathrm{~m}$ ), dans des terrains difficiles et

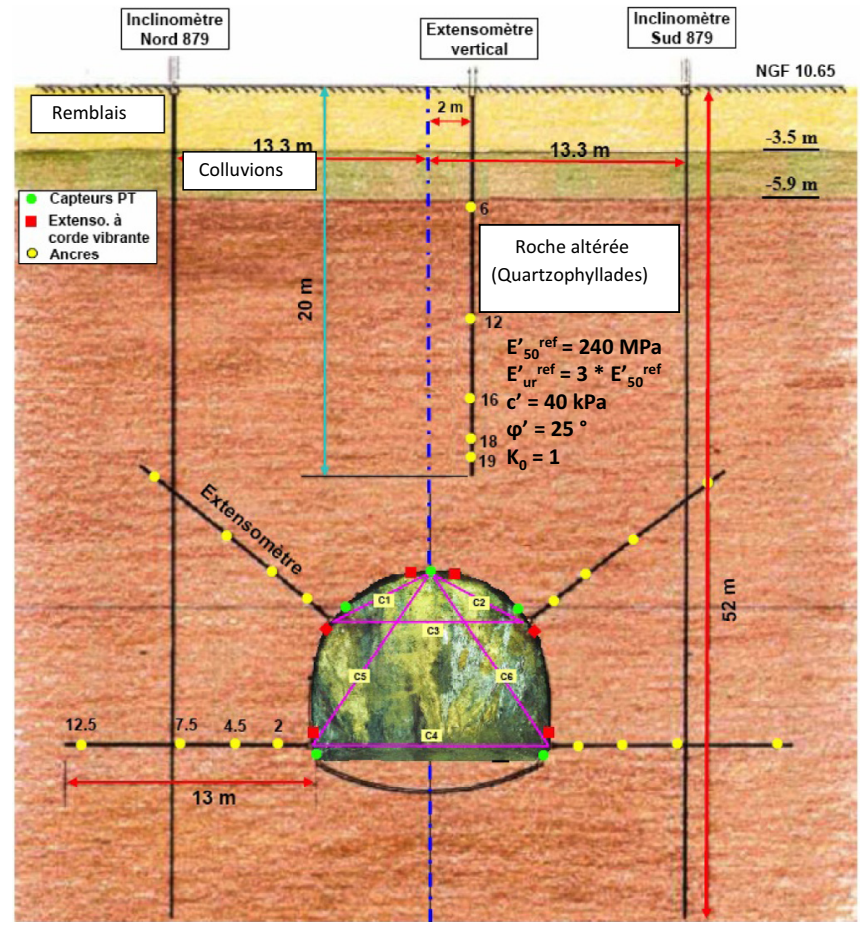

Fig. 1. Section instrumentée - Tunnel sud de Toulon.

Fig. 1. Monitoring section - South Toulon tunnel.

de nature hétérogène. Le tunnel a été creusé par méthode conventionnelle avec présoutènement par forepoling en voûte et boulonnage au front de taille. Un système d'auscultation conséquent en surface, basé sur une série de théodolites automatiques qui enregistraient les déplacements du sol et des bâtiments avec une fréquence de mesure élevée, a été mis en place et suivi pendant toute la durée de réalisation du tunnel. Le phasage des travaux ainsi que le système de pré-renforcement ont été continuellement adaptés en fonction principalement de la couverture, des sols rencontrés et des mesures enregistrées en surface et en souterrain (Janin et al., 2011). Dans le cadre de la thèse, deux sections instrumentées ont été mises en place. Les mesures recueillies ont constitué une base de données pour les rétro-analyse numériques (Janin, 2012) (Fig. 1).

L'approche 3D s'est révélée capable de simuler le phénomène tridimensionnel d'interaction entre l'excavation, les renforcements, la réaction du sol et du soutènement (Janin et al., 2015). En particulier, les enseignements suivants ont pu être formalisés :

- la nécessité de la prise en compte de la non-linéarité du comportement réel du sol, de la distinction entre module en déchargement/rechargement et module en premier chargement et de l'évolution des modules en fonction des contraintes. Au contraire de modèles de comportement élastiques parfaitement plastiques, conduisant à une sousestimation du tassement maximal, à une surestimation de la largeur de la cuvette et à une surestimation du soulèvement en radier, phénomènes bien connus (Hejazi et al., 2008), et nécessitant le recours à des artefacts tels que la subdivision $\mathrm{du}$ massif en sous-couches fictives pour pouvoir se caler sur les mesures enregistrées, le modèle de comportement 


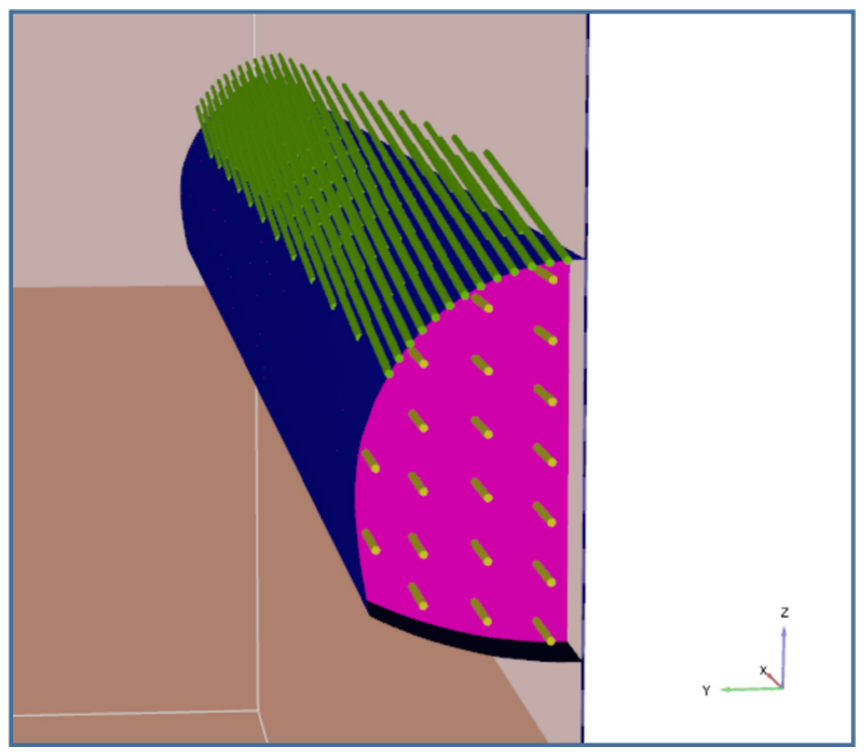

Fig. 2. Modélisation 3D, présoutènement et soutènement - Tunnel sud de Toulon.

Fig. 2. 3D model, presupport and lining - South Toulon tunnel.

Hardening Soil Model (HSM), implémenté dans Plaxis, a permis de reproduire numériquement, de façon très satisfaisante, les mouvements du massif enregistrés avec différents instruments de mesures (cibles de tassements en surface, extensomètres verticaux et radiaux, inclinomètres);
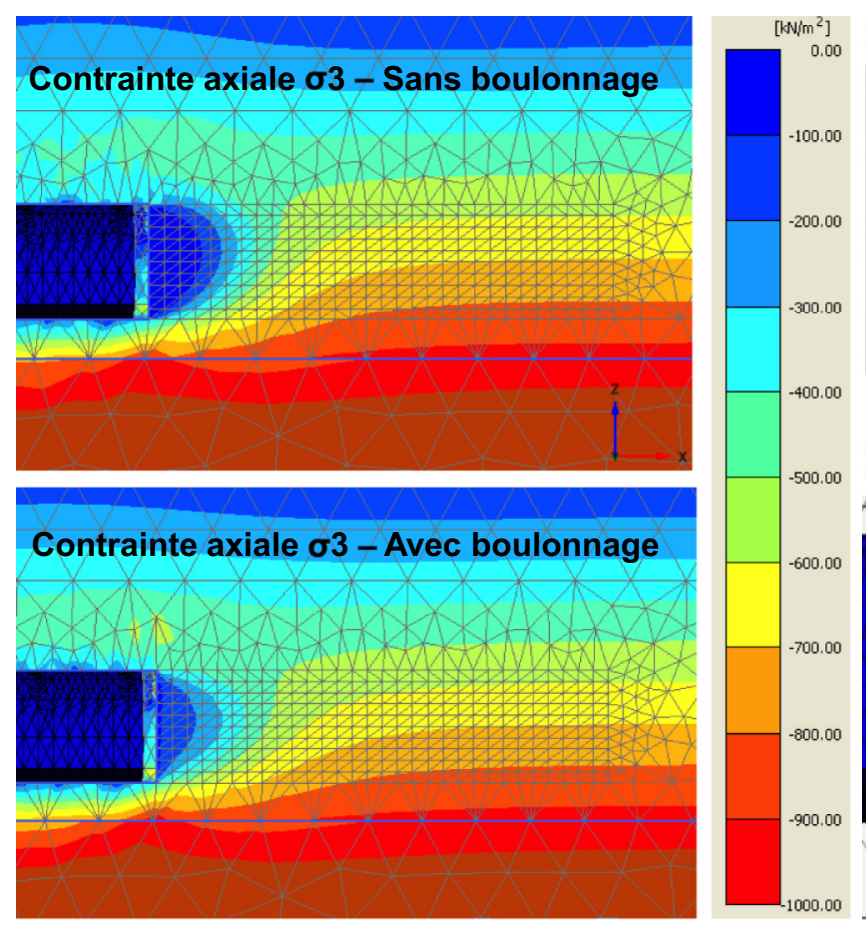

(a)
- l'importance d'une modélisation par discrétisation (modélisation explicite) des éléments de présoutènement: boulonnage au front et forepoling (Fig. 2). Comme montré, en effet, par plusieurs auteurs, les approches en homogénéisation surestiment souvent l'effet de ces renforcements vis-à-vis des déformations dans le massif (Volkmann et al., 2006; Eclaircy-Caudron et al., 2006; Dias, 2011). Dans notre cas, la modélisation explicite des inclusions et du comportement à l'interface sol-inclusion a permis de mettre en évidence le rôle majeur du boulonnage au front dans le confinement du noyau à l'avant du front (Fig. 3), en cohérence avec les principes de la méthode ADECO-RS (Analysis of controlled deformation in rocks and soils) de Lunardi (2008);

- la nécessité d'adopter l'approche 3D permettant de mieux se rapprocher du réel phasage des travaux, en éliminant la variable « taux de déconfinement », souvent choisie de façon arbitraire et ayant un impact important sur les résultats. En particulier, l'approche tridimensionnelle s'est révélée non seulement capable de simuler la tridimensionnalité du champ des déformations généré autour du tunnel (Fig. 4 et 5), mais également de modéliser correctement le chargement du soutènement (Fig. 6). Dans les calculs bidimensionnels en déformations planes, au contraire, il est souvent nécessaire de choisir deux taux de déconfinement différents, selon que l'on cherche à se caler sur les déplacements mesurés ou sur les efforts mesurés dans le soutènement (Möller et Vermeer, 2008). L'approche 2D n'est pas capable, en effet, de représenter les phénomènes $3 \mathrm{D}$ complexes d'effets voûtes longitudinaux et de chargement du soutènement, en particulier à proximité du front de taille.
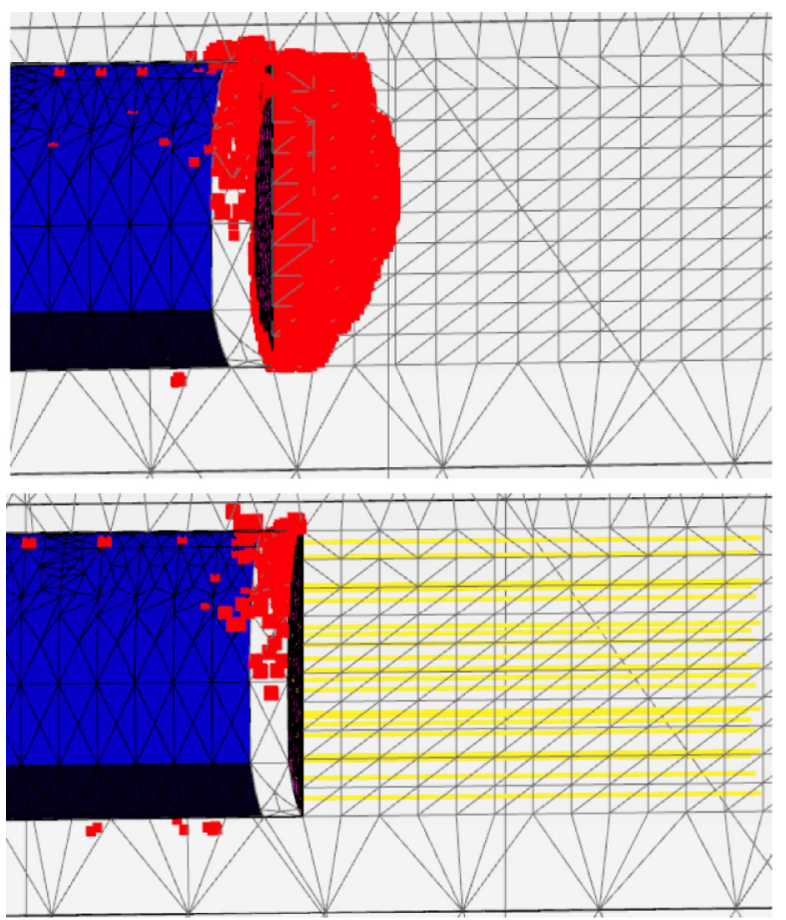

(b)

Fig. 3. Modélisation 3D, effet du boulonnage au front sur la contrainte axiale $\sigma_{3}$ (a) et sur l'état de rupture du massif(b) - Tunnel sud de Toulon. Fig. 3. 3D model, face bolts effects on axial stresses $\sigma_{3}(a)$ and on soil plastification (b) - South Toulon tunnel. 


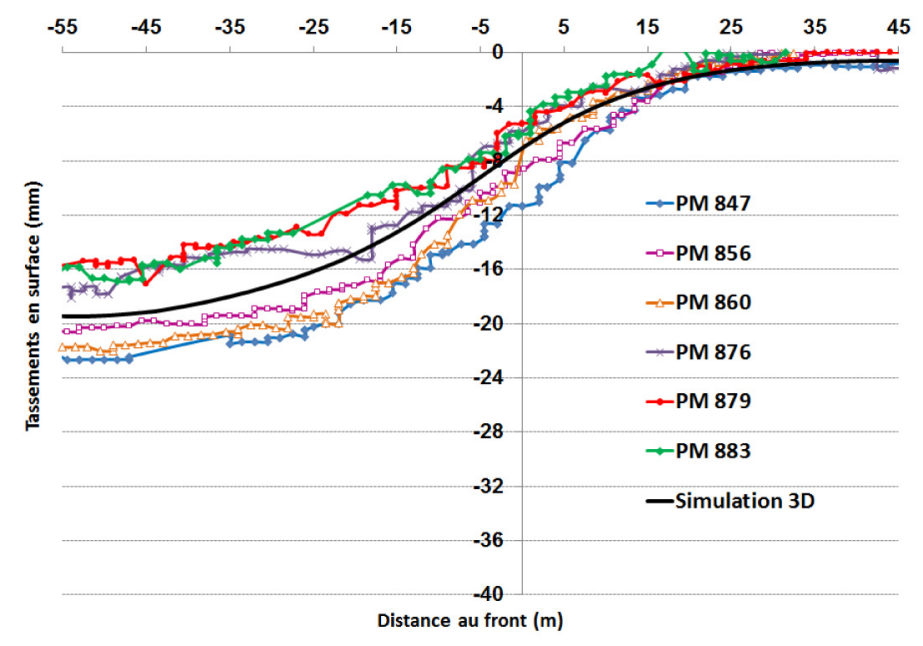

(a)

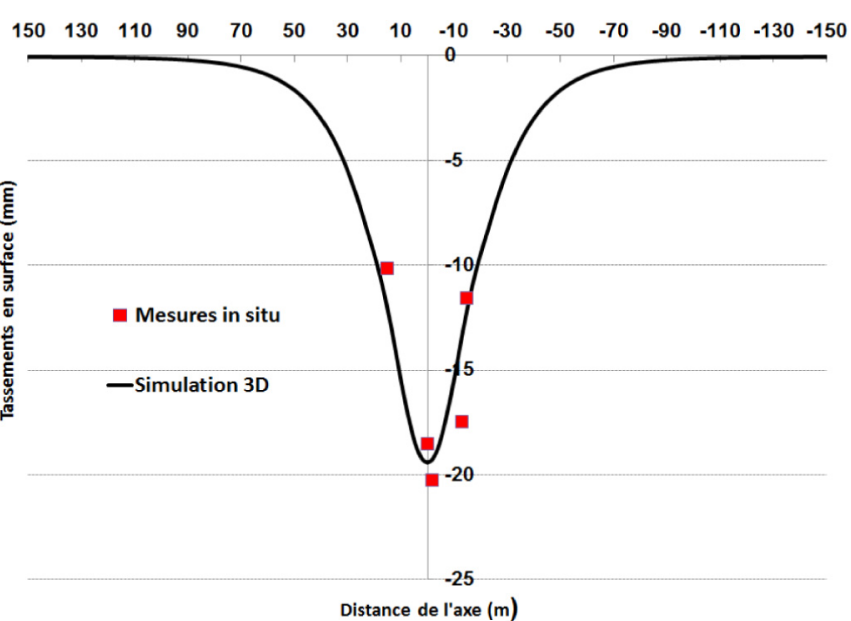

(b)

Fig. 4. Profil en long des tassements (a) et cuvette transversale (b) - comparaison 3D/mesures in situ - Tunnel sud de Toulon.

Fig. 4. Longitudinal settlement profile (a) and settlement in the transverse section (b) - 3D comparison/in situ measurements - South Toulon tunnel.

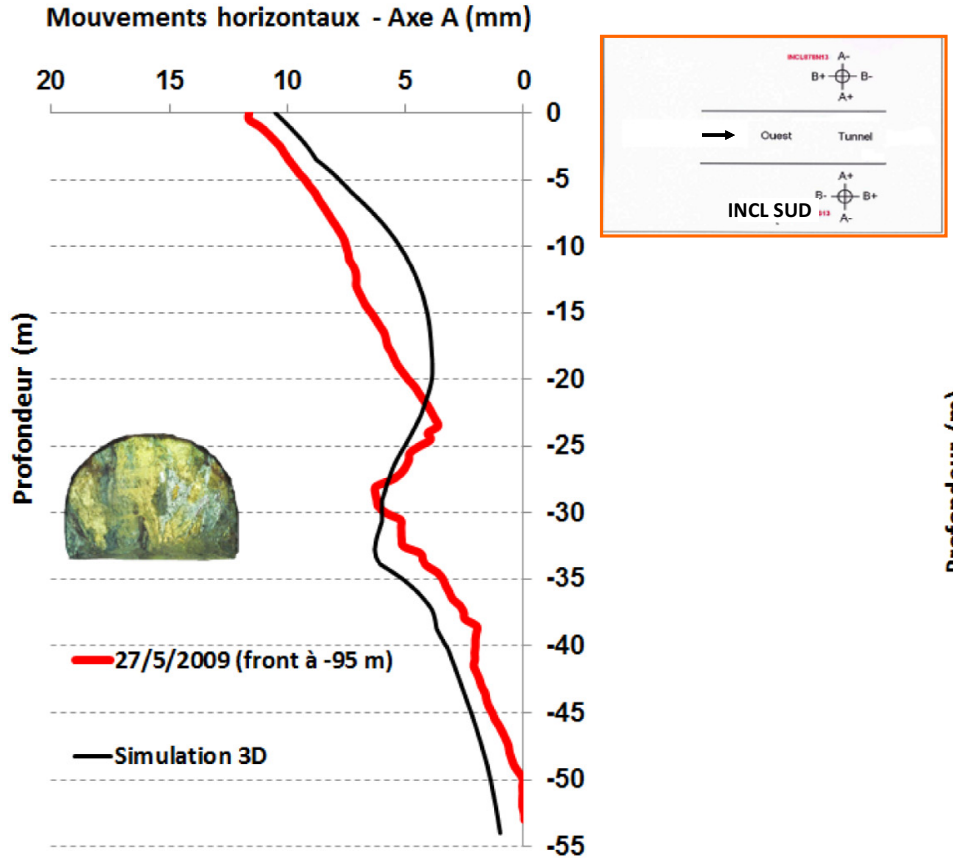

(a)

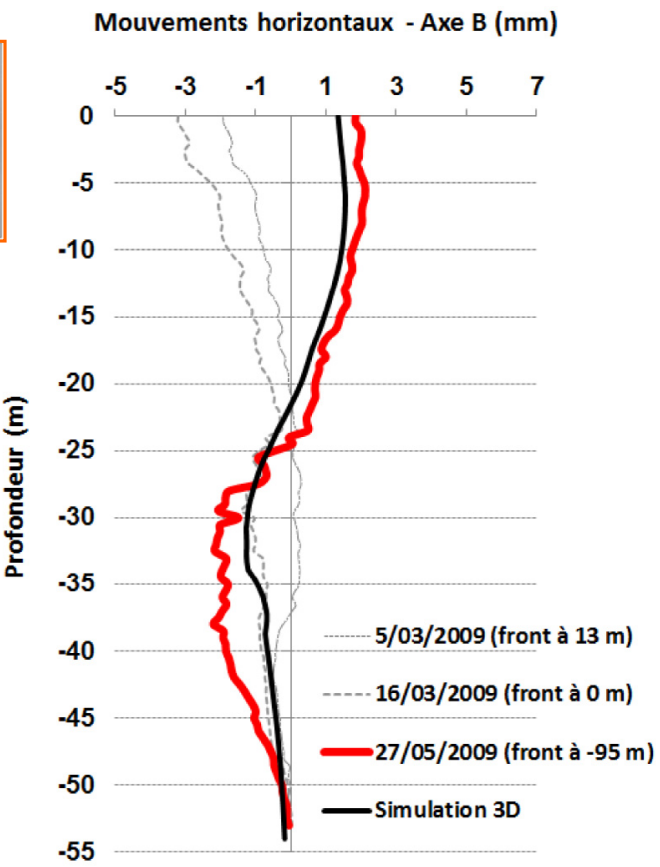

(b)

Fig. 5. Déplacements inclinométriques selon l'axe A (a) et l'axe B (b) - comparaison 3D/mesures in situ - Tunnel sud de Toulon. Fig. 5. Inclinometer movements along axis $A(a)$ and axis $B(b)-3 D$ comparison/in situ measurements - South Toulon tunnel.

\section{Excavation multiphases: chambre de démontage d'un tunnelier}

Lors de la réalisation d'ouvrages souterrains présentant une section importante mais une longueur limitée, le terrassement en méthode traditionnelle, en section divisée, est souvent adopté. Dans ces situations, l'approche bidimen- sionnelle peut conduire à une surestimation des déformations, susceptible de remettre en cause la faisabilité du projet. Une modélisation 3D permet alors de simuler plus correctement le phasage réel des travaux, de prendre en compte le report de charge sur les parties déjà réalisées et de mieux modéliser les ouvrages existants.

Cela a été le cas, par exemple, de l'étude concernant la réalisation de la chambre de démontage d'un tunnelier dans 


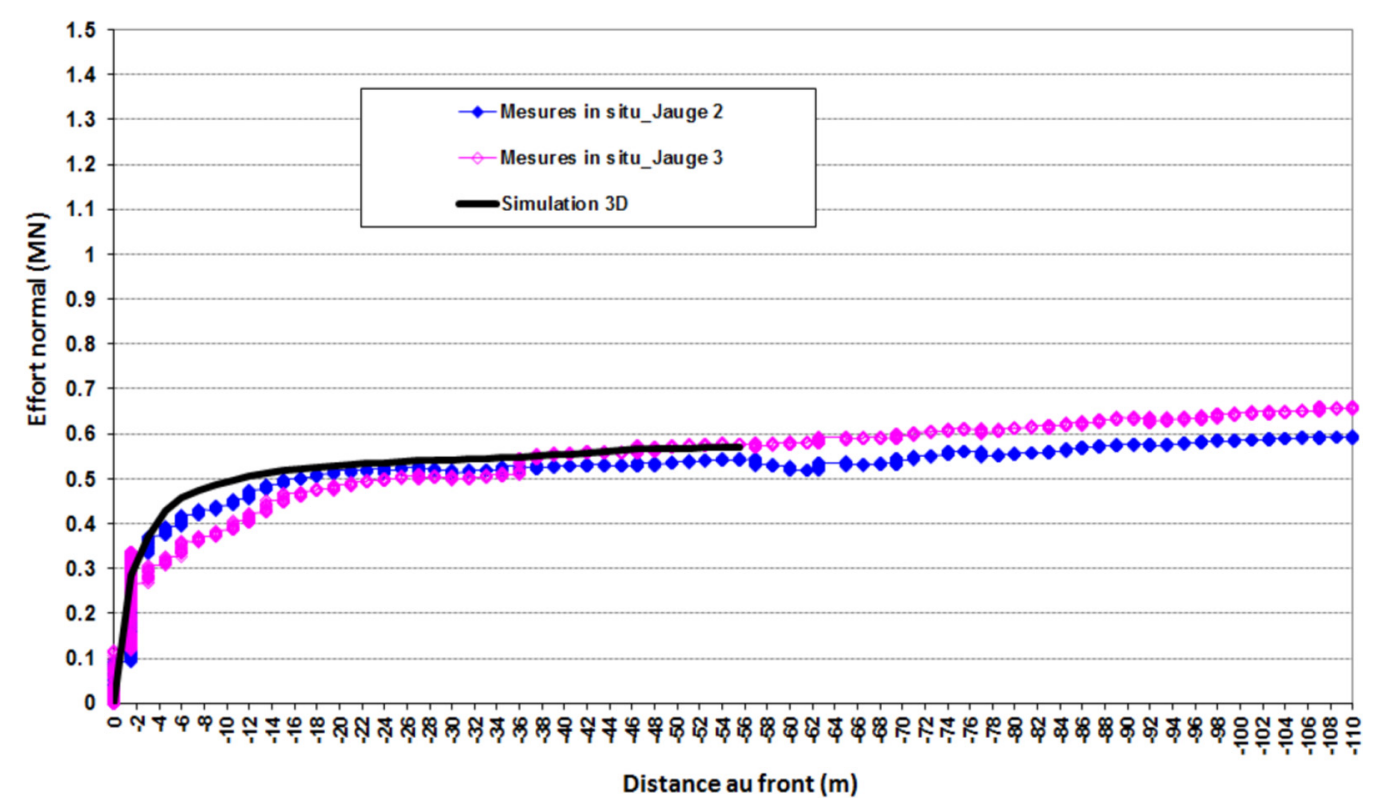

Fig. 6. Évolution de l'effort normal dans les cintres - comparaison 3D/mesures in situ - Tunnel sud de Toulon.

Fig. 6. Evolution of the normal force on the rib - 3D comparison/in situ measurements - South Toulon tunnel.

le $9^{\mathrm{e}}$ arrondissement de Paris, à une dizaine de mètres sous des bâtiments haussmanniens sensibles, dans le cadre du prolongement nord de la ligne 14 du métro parisien (Janin et al., 2014). La chambre est composée de deux parties principales (Fig. 7):

- la chambre «à grande section », dans laquelle le tunnelier doit être démonté (section excavée de l'ordre de $90 \mathrm{~m}^{2}$ et longueur de $5,4 \mathrm{~m}$ );

- la partie tronconique, nommée entonnoir, qui relie la section du tunnel existant à la chambre de démontage, ayant une longueur de 4,3 m.

Lors de premières études, une approche numérique $2 \mathrm{D}$ en déformations planes avait été adoptée. Celle-ci concluait à un tassement maximal au niveau des bâtiments de $25 \mathrm{~mm}$, valeur bien au-delà du seuil de $10 \mathrm{~mm}$ à respecter. Suite à une optimisation de la géométrie de la chambre, il a été ainsi décidé avec la Maîtrise d'œuvre (MOE) du projet (Systra) d'étudier le problème par une approche de calcul 3D. Le phasage complexe de réalisation de l'ouvrage a pu être simulé, en éliminant la variable du taux de déconfinement, dont le choix est très délicat dans ce genre de projet. Le creusement de l'ouvrage, en section divisée, dans la formation des Marnes et Caillasses $\left(E^{\prime}{ }_{50}{ }^{\text {ref }}=\right.$ $\left.200 \mathrm{MPa}, E_{\text {ur }}^{\prime}{ }_{\text {ref }}=3 \times E^{\prime}{ }_{50}^{\text {ref }}, c^{\prime}=30 \mathrm{kPa}, \varphi^{\prime}=27\right)$, a été conçu de la façon suivante :

- excavation du four avec des passes de $1 \mathrm{~m}$ environ et mise en place d'un soutènement provisoire composé de cintre HEB (European wide flange beams) et béton projeté (Fig. 8a);

- excavation des abattages latéraux, selon les mêmes passes, avec prolongement du soutènement provisoire composé de cintre HEB avec pattes d'éléphant et béton projeté (Fig. 8b) ;

- réalisation du revêtement de la voûte et d'une partie du tympan final;

- excavation du merlon central et boulonnage au front de la section demi-inférieure;
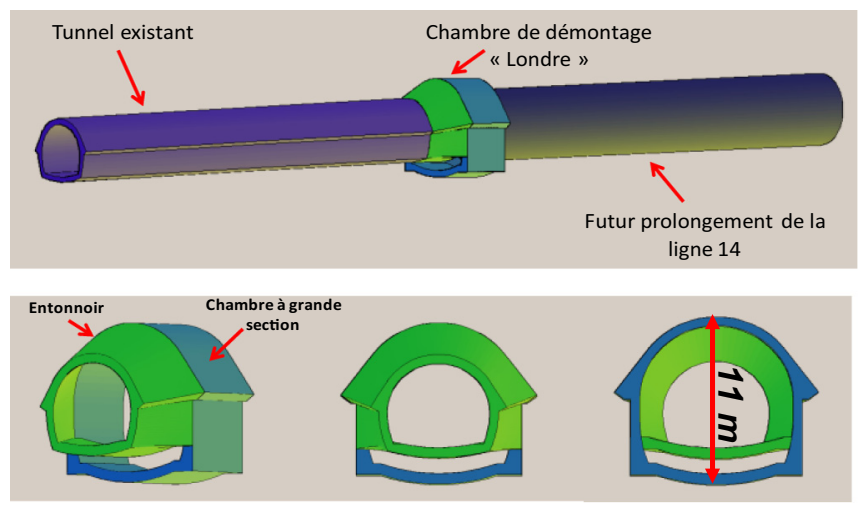

Fig. 7. Géométrie de la chambre de démontage du tunnelier.

Fig. 7. TBM dismantling cavern geometry. a)
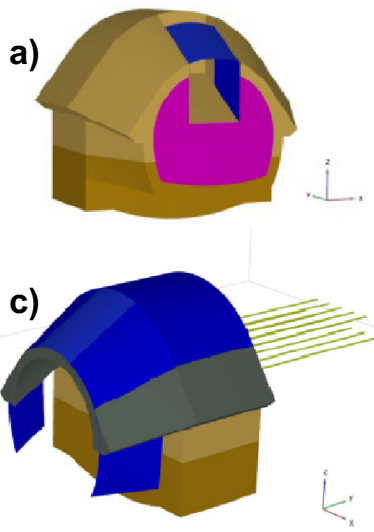
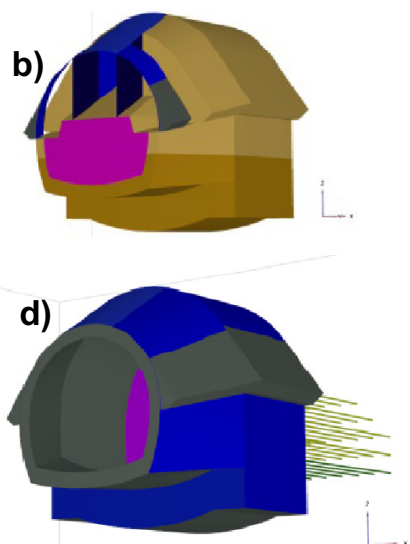

Fig. 8. Simulation $3 \mathrm{D}$ des étapes de réalisation de la chambre de démontage.

Fig. 8. 3D simulation of dismantling cavern realization process. 


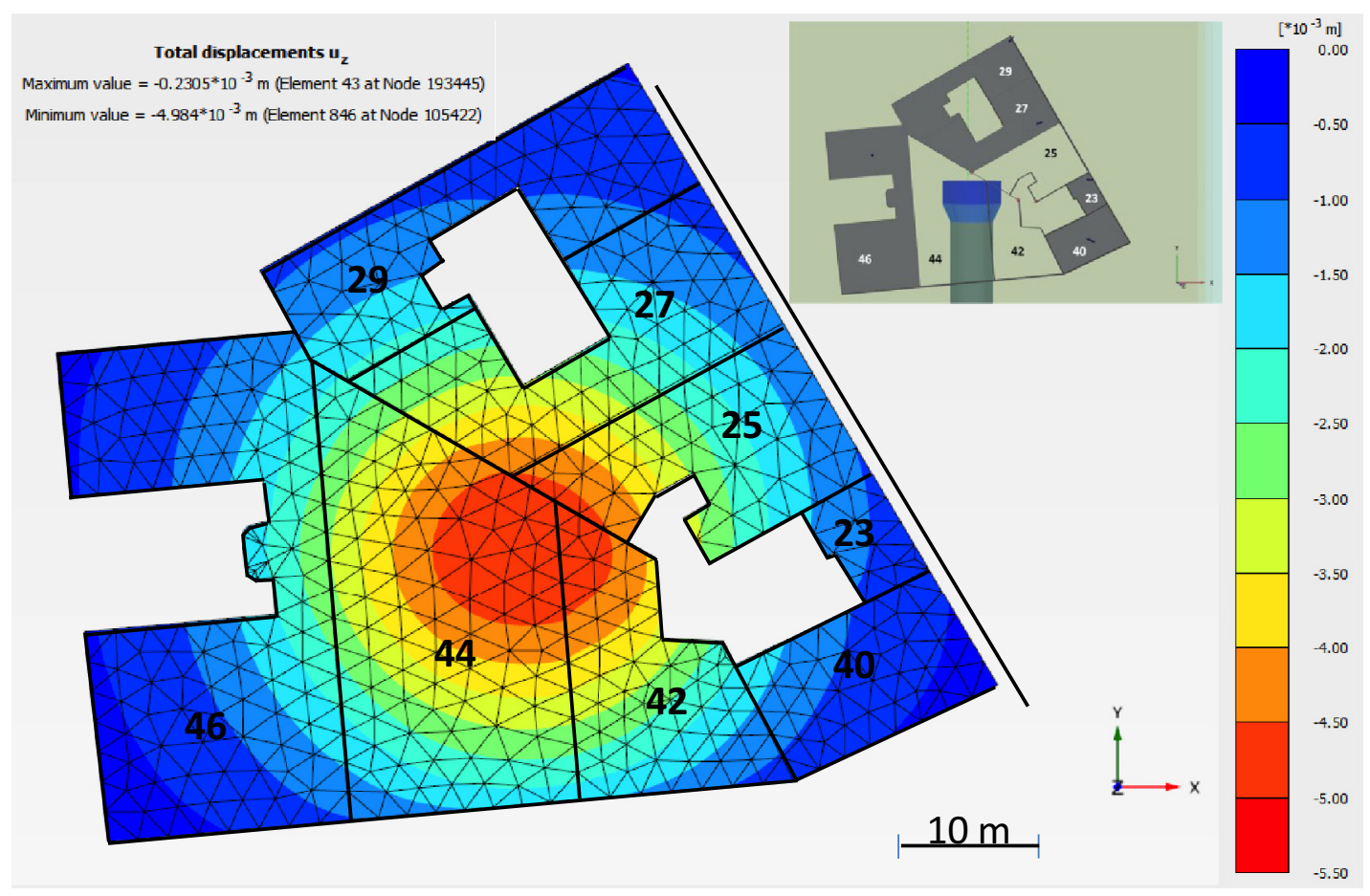

Fig. 9. Tassements finaux sous les fondations des bâtiments.

Fig. 9. Final building settlements.

- excavation de la première partie de la section demiinférieure (Fig. 8c) et, ensuite, réalisation du revêtement; - excavation de la deuxième partie de la section demiinférieure et finalisation du revêtement (Fig. 8d).

Différents résultats ont pu être analysés, tels que les tassements au niveau des bâtiments, les déplacements dans le massif, la convergence et les efforts dans les soutènements, permettant de définir un dispositif d'auscultation adapté ainsi que les seuils à utiliser dans le cadre de la méthode observationnelle. À ce propos, l'approche 3D permet d'accéder à des résultats non seulement probablement plus corrects mais aussi plus «complets». Par rapport à une modélisation bidimensionnelle, on peut, par exemple, modéliser l'emprise correcte des différents bâtiments et identifier ceux qui seront le plus concernés par des tassements absolus et différentiels significatifs (Fig. 9). Finalement, la méthodologie de construction de la chambre de démontage a pu être justifiée, avec des tassements des bâtiments inférieurs au seuil de $10 \mathrm{~mm}$.

\section{Interaction avec les ouvrages existants: adaptation d'une station de métro}

Dans les études des ouvrages souterrains, on doit souvent faire face à la problématique des interactions entre les nouvelles structures à réaliser et celles déjà existantes. La modélisation tridimensionnelle devient ainsi un outil indispensable pour tenir compte de la géométrie complexe du problème et de l'historique des contraintes.

Une étude réalisée récemment la Régie autonome des transports parisiens (RATP) (MOE du projet) a concerné la réalisation d'accès secondaires à la station existante « Mairie des
Lilas », sur la ligne 11 du métro parisien. Les travaux consistent en la réalisation de puits blindés depuis la surface, à partir desquels des galeries souterraines seront excavées pour venir recouper les ouvrages existants (salle des billets, rameaux, station souterraine) et créer ainsi des sorties supplémentaires. Les enjeux et les objectifs de l'étude, demandés par la RATP, étaient multiples : concevoir le phasage des travaux, justifier le soutènement provisoire des ouvrages, évaluer les effets de la construction des nouveaux accès sur les ouvrages existants en souterrain (station et ligne de métro) et sur les bâtiments et la voirie en surface et définir, enfin, un dispositif d'auscultation.

Étant donné la géométrie du problème à traiter, une approche numérique tridimensionnelle nous est apparue essentielle pour étudier correctement la complexité des interactions sol-structure entre les travaux et les ouvrages existants et répondre aux différents objectifs demandés. Une modélisation numériques 3D complexe a été ainsi réalisée (Fig. 10). Elle a permis de simuler dans le détail les ouvrages existants (en souterrain et en surface) ainsi que le phasage des travaux des accès supplémentaires.

La simulation numérique a permis d'analyser les tassements générés en surface et au niveau des bâtiments (Fig. 11a), les déplacements induits dans les ouvrages souterrains existants (Fig. 11b) et les efforts dans les soutènements provisoires des nouveaux accès.

L'analyse des résultats a mis en évidence, en particulier, que la cause principale des tassements est le creusement de la galerie d'accès à la salle des billets (Fig. 10 et 11). Étant donné sa faible couverture (rapport couverture/diamètre proche de 1), un suivi attentif devra être mis en place lors de sa réalisation. À ce propos, l'approche 3D nous a donné accès non seulement aux convergences mais également à l'extrusion générée au front. Celle-ci représente une donnée très importante à suivre lors des 


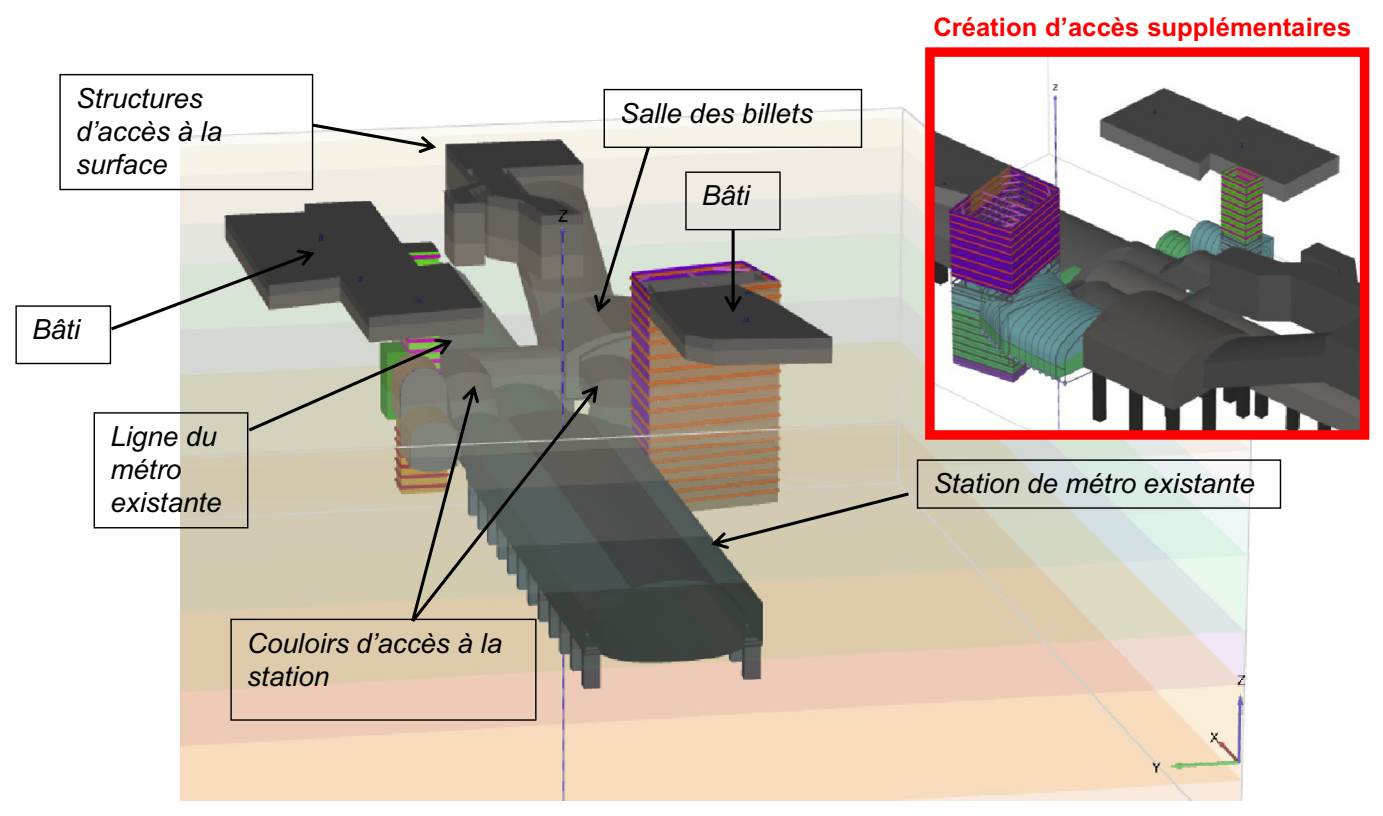

Fig. 10. Simulation numérique $3 \mathrm{D}$ de la réalisation d'accès supplémentaires à une station existante du métro parisien.

Fig. 10. 3D numerical simulation of additional access realization to an existing station of Paris metro.

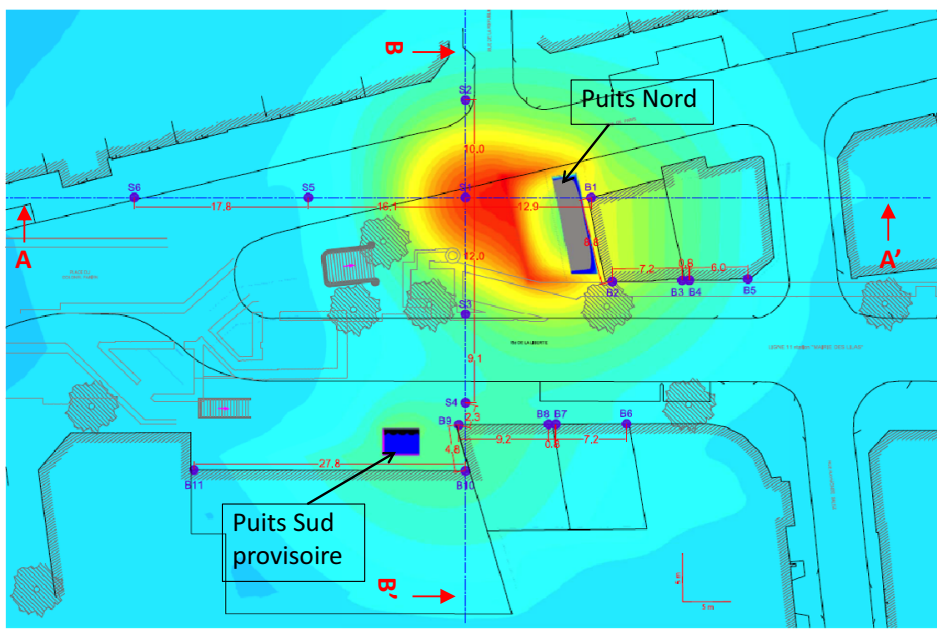

(a)

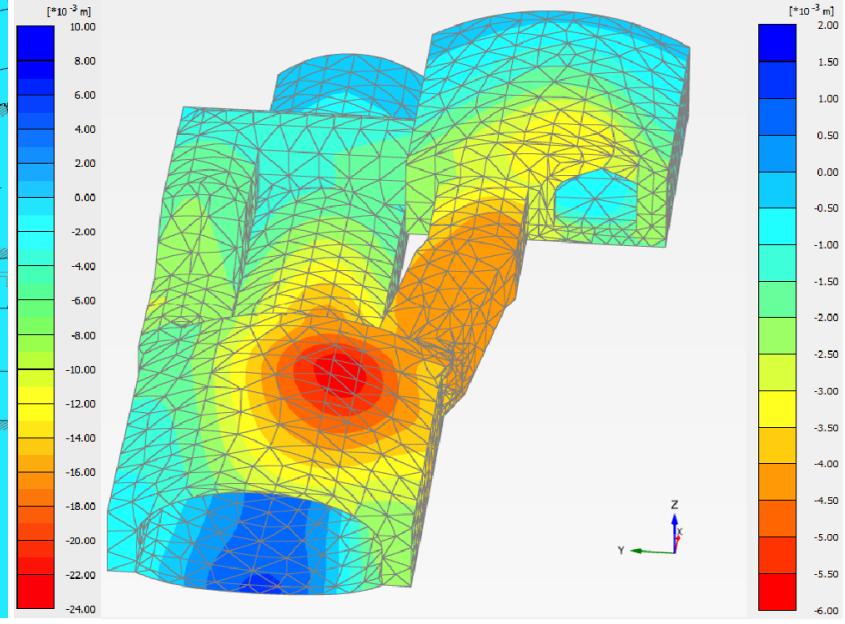

(b)

Fig. 11. Tassements en surface avec positionnement des cibles d'auscultation (a) et déplacements verticaux engendrés dans les ouvrages souterrains (b).

Fig. 11. Surface settlements with positioning of settlement auscultation (a) and vertical movements generated in underground structures (b).

travaux parce que, dans le cas d'une galerie peu profonde, elle est strictement liée aux tassements qu'on va générer en surface.

L'exploitation de ces différents résultats nous a enfin permis de concevoir, lors de la phase DCE (Dossier de consultation des entreprises), un dispositif d'auscultation, qui sera adopté lors du chantier dans le cadre de la mise en place de la méthode observationnelle.

\section{Excavation en front mixte : future L15 du Grand Paris}

L'excavation d'un tunnel en front mixte, dans des formations présentant un fort contraste de rigidités et de résistances, rend très délicat le choix d'un taux de déconfinement représentatif des effets de voûte qui se développent longitudinalement autour de l'excavation. Pour le calcul du taux de déconfinement, l'ingénieur, dans ce cas, se pose toujours la question du choix des caractéristiques mécaniques: caractéristiques homogénéisées ou bien, selon une approche pessimiste, considérer celles les plus défavorables. En particulier, dans le cas où une des deux formations encaissantes présente des faibles caractéristiques de résistance au cisaillement par rapport aux contraintes en place (valeur élevée du nombre de stabilité $N=2 \sigma_{0} / R c$ ), ce choix est particulièrement délicat, parce qu'une faible variation du taux peut amener à des différences significatives en termes de 

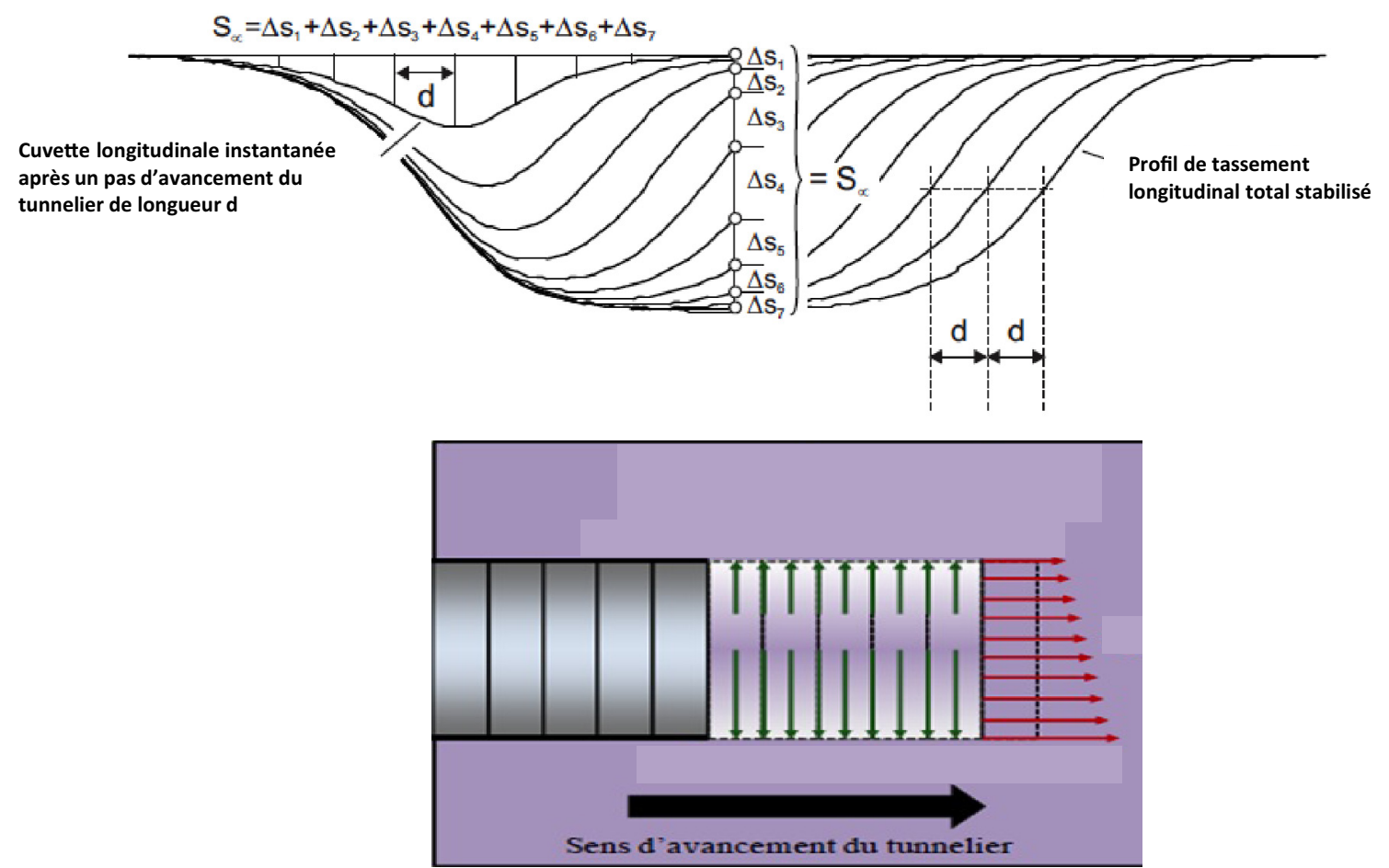

Fig. 12. Principe de détermination du tassement longitudinal stabilisé avec la méthode de la cuvette instantanée.

Fig. 12. Principle of determination of the longitudinal stabilized settlement profile with the method of instantaneous settlement trough.

déplacements dans le massif et de tassements en surface (ceci en fonction de l'étendue de la zone plastique générée).

Cette problématique s'est posée lors des études pour le projet de la future ligne 15 sud du projet du Grand Paris Express. Dans le sous-tronçon, qui va environ de ChâtillonMontrouge à Arcueil-Cachan, le tunnelier se trouvera souvent dans une configuration en front mixte calcaire grossier/argiles plastiques. Un des objectifs principaux des études réalisées a été de définir la profondeur du tracé et la pression de confinement $\left(P_{\text {confinement }}\right)$ de manière à respecter les seuils de tassements définis au niveau des bâtiments présents en surface. Une réflexion sur la méthodologie de calcul à adopter a été ainsi menée.

Comme déjà énoncé ci-dessus, l'approche de calcul en déformations planes, basée sur la méthode convergenceconfinement, montre, dans ce cas, ses limites. Dans le cas de creusement au tunnelier avec une pression de stabilisation au front, il est d'usage de corriger le taux de déconfinement classiquement calculé par la pression de confinement du tunnelier rapportée à la contrainte initiale in situ $\left(\sigma_{0}\right)$, selon la formule ci-dessous :

$$
\lambda_{\text {corriag }}=\lambda_{d} \cdot\left(1-\frac{P_{\text {confinement }}}{\sigma_{0}}\right)
$$

Les limites principales de cette approche sont les suivantes :

- on ne respecte pas souvent les hypothèses de base de la méthode convergence-confinement: dans notre cas d'étude, par exemple, le milieu n'est pas homogène et l'état de contraintes initial est loin d'être isotrope;

- on vient corriger le taux de déconfinement par une valeur fixe de la pression de confinement et de $\sigma_{0}$. Outre la problématique du choix de la valeur de la contrainte initiale, la pression de confinement peut également varier significativement entre la clé et le radier du tunnel, ceci d'autant plus que les tunnels sont de grands diamètres; - on vient appliquer une valeur unique du taux déconfinement sur tout le contour de l'excavation: des calculs comparatifs $3 \mathrm{D} / 2 \mathrm{D}$ montrent que cette condition est très rarement respectée, même pour des configurations plus simples (front homogène);

- il est très compliqué de simuler les différentes étapes du processus d'avancement d'un tunnelier par une approche de calcul 2D.

Pour faire face à ces différentes problématiques, on a mis en œuvre une approche numérique 3D «simplifiée» (sur le code de calcul PLAXIS 3D). Cette approche se base sur le concept de «cuvette longitudinale instantanée de tassement», déjà développé par d'autres auteurs pour des tunnels réalisés en méthode traditionnelle (Serratrice et Magnan, 2002) et au tunnelier (Möller, 2006). Le concept repose sur les idées suivantes (Fig. 12):

- le processus de creusement du tunnel est une succession d'opérations, répétées à chaque pas d'avancement, qui a une longueur égale à celle des voussoirs ;

- à chaque avancement du tunnelier, on génère une cuvette de tassement en surface, dite «instantanée», qui sera la même pour la phase suivante, mais décalée le long de l'axe d'une distance égale au pas d'avancement;

- en sommant les cuvettes instantanées de tassement, on peut ainsi déduire le profil longitudinal de tassement et la cuvette transversale stabilisée.

La comparaison entre des calculs «complets» (où l'avancement du tunnelier est simulé sur plusieurs phases 
permettant d'atteindre un état stationnaire des déplacements à l'arrière du front) et des calculs avec la «méthode 3D simplifiée », présentée ci-dessus, a montré une très bonne concordance entre les deux approches (Dano, 2014).

Les principaux avantages de la «méthode 3D simplifiée » sont les suivants :

- une prise en compte «directe» de la problématique 3D, en éliminant la variable du taux de déconfinement;

- une modélisation explicite et distincte des différents facteurs qui interviennent dans le processus d'une excavation mécanisée au tunnelier pressurisé et qui conditionnent directement les déplacements générés par l'excavation (Clough et al., 1985; Kastner et al., 1996; Mair et Taylor, 1997) : pression de confinement au front et éventuellement autour de la jupe, présence de la jupe et de sa conicité, pression du mortier de bourrage, voussoirs...);

- une réduction très significative des temps de calcul par rapport à une approche $3 \mathrm{D}$ complète d'avancement du tunnelier;

- un accès à plus de résultats «utiles» par rapport à une approche 2D, tels que la plastification du massif à l'avant du front et autour de la jupe, le profil en long des tassements (avec également le tassement différentiel longitudinal), les profils de convergence autour de l'excavation (y compris les pré-convergences), l'extrusion au front, etc.

En revanche, cette méthode ne permet pas d'évaluer les efforts dans le revêtement.

On présente ci-après les résultats d'un exemple d'application de la «méthode 3D simplifiée». Il s'agit de l'excavation d'un tunnel, ayant un diamètre de $10 \mathrm{~m}$, par un tunnelier à pression de terre, mettant en place une pression de confinement de $300 \mathrm{kPa}$ au front et autour de la jupe, avec un gradient vertical dans la chambre de $15 \mathrm{kPa} / \mathrm{m}$. L'excavation, sous une couverture de $35 \mathrm{~m}$, se fait en front mixte calcaire grossier $\left(E^{\prime}=800 \mathrm{MPa}, c^{\prime}=100 \mathrm{kPa}\right.$ et $\left.\phi^{\prime}=40^{\circ}, K_{0}=0,35\right)$ et argiles plastiques $\left(E_{\mathrm{CT}}=75 \mathrm{MPa}, C u=150 \mathrm{kPa}\right.$ et $\left.\phi_{\mathrm{u}}=0^{\circ}, K_{0}=0,75\right)$.

La Figure 13 montre les profils de déplacement radial «Ur» dans quatre différents points autour du profil excavé. Ainsi que suspecté, la méthode aboutit à des allures de déplacements bien différentes selon la position du point considéré et le type de sol encaissant. En particulier, il est intéressant de noter que dans les argiles plastiques, dans la zone à l'avant du front, on obtient des déplacements radiaux divergents. D'après une analyse de l'évolution des contraintes autour de l'excavation, ceci est lié à une augmentation de la contrainte moyenne à un diamètre environ de distance à l'avant du front. Le phénomène des effets voûtes, qui se développent longitudinalement autour de l'excavation, se montre ainsi bien complexe dans ce genre de configuration.

A partir du graphe présenté sur la Figure 13, il est ainsi possible d'obtenir les déplacements radiaux du sol à la sortie de la jupe (de $10 \mathrm{~m}$ de long dans ce cas d'étude), avant la pose des voussoirs. Il est intéressant ensuite de réaliser une modélisation en déformations planes (avec les mêmes hypothèses géométriques, géotechniques et relatives au tunnelier) et faire varier le taux de déconfinement pour retrouver ces mêmes déplacements radiaux. Les résultats de cette étude sont montrés dans le Tableau 1. Comme déjà énoncé, il apparaît clairement que le taux de déconfinement obtenu varie en fonction du point considéré autour de l'excavation.

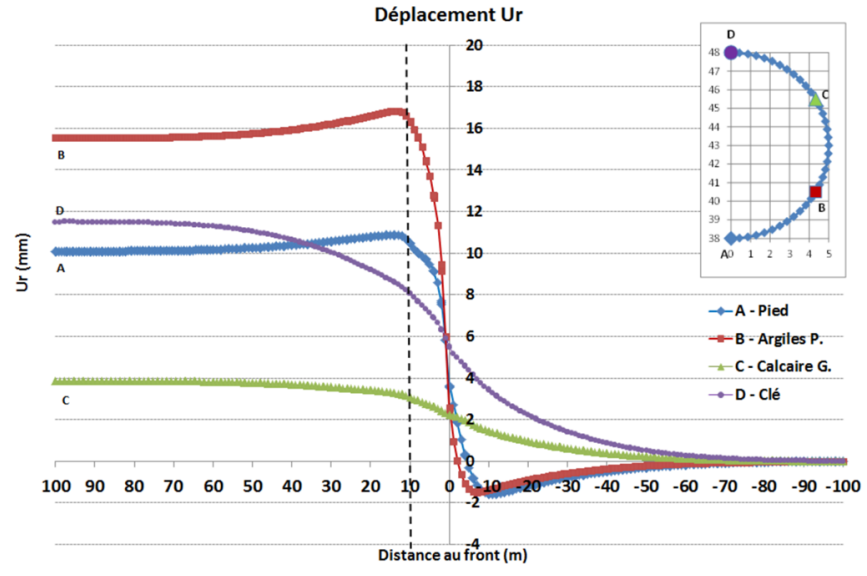

Fig. 13. Profils du déplacement radial en fonction de la distance au front (méthode 3D simplifiée).

Fig. 13. Radial displacement profiles versus distance to face (3D simplified method)

Tableau 1. Taux de déconfinement calculé en sortie de jupe. Table 1. Stress release coefficient calculated at the end of shield.

\begin{tabular}{lll}
\hline Point & Ur à l'arrière de la jupe $(\mathrm{mm})$ & $\lambda$ arrière jupe $(x / r=2)$ \\
\hline A & 10,5 & 0,53 \\
B & 16,3 & 0,40 \\
C & 3 & 0,32 \\
D & 8 & 0,39 \\
\hline
\end{tabular}

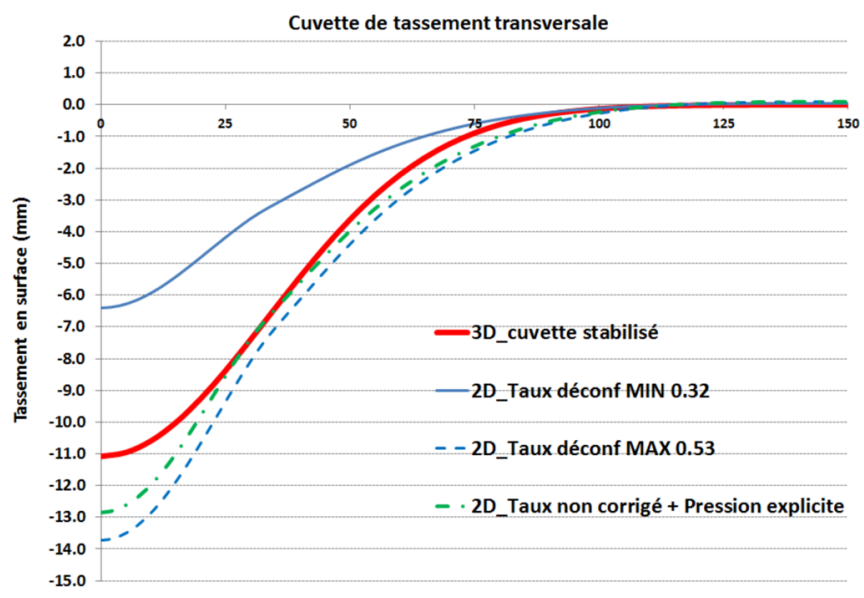

Fig. 14. Cuvette transversal de tassement-comparaison $2 \mathrm{D}$ versus $3 \mathrm{D}$.

Fig. 14. Transversal settlement profile $-2 D$ versus $3 D$ comparison.

Si ensuite on fait l'exercice d'injecter dans un calcul 2D en déformations planes le taux de déconfinement minimale ou maximale déterminé (Fig. 14), on constate que la cuvette transversale de tassement calculée s'écarte de celle obtenue avec l'approche 3D (de 30 à $40 \%$ d'écart dans ce cas d'étude). D'après notre expérience, cet écart tend à augmenter si le front de taille rentre davantage dans les argiles plastiques et si la couverture augmente. Il est possible de mieux se caler sur la cuvette obtenue en $3 \mathrm{D}$, en prenant en compte un taux de déconfinement non corrigé et en simulant explicitement la pression de confinement 


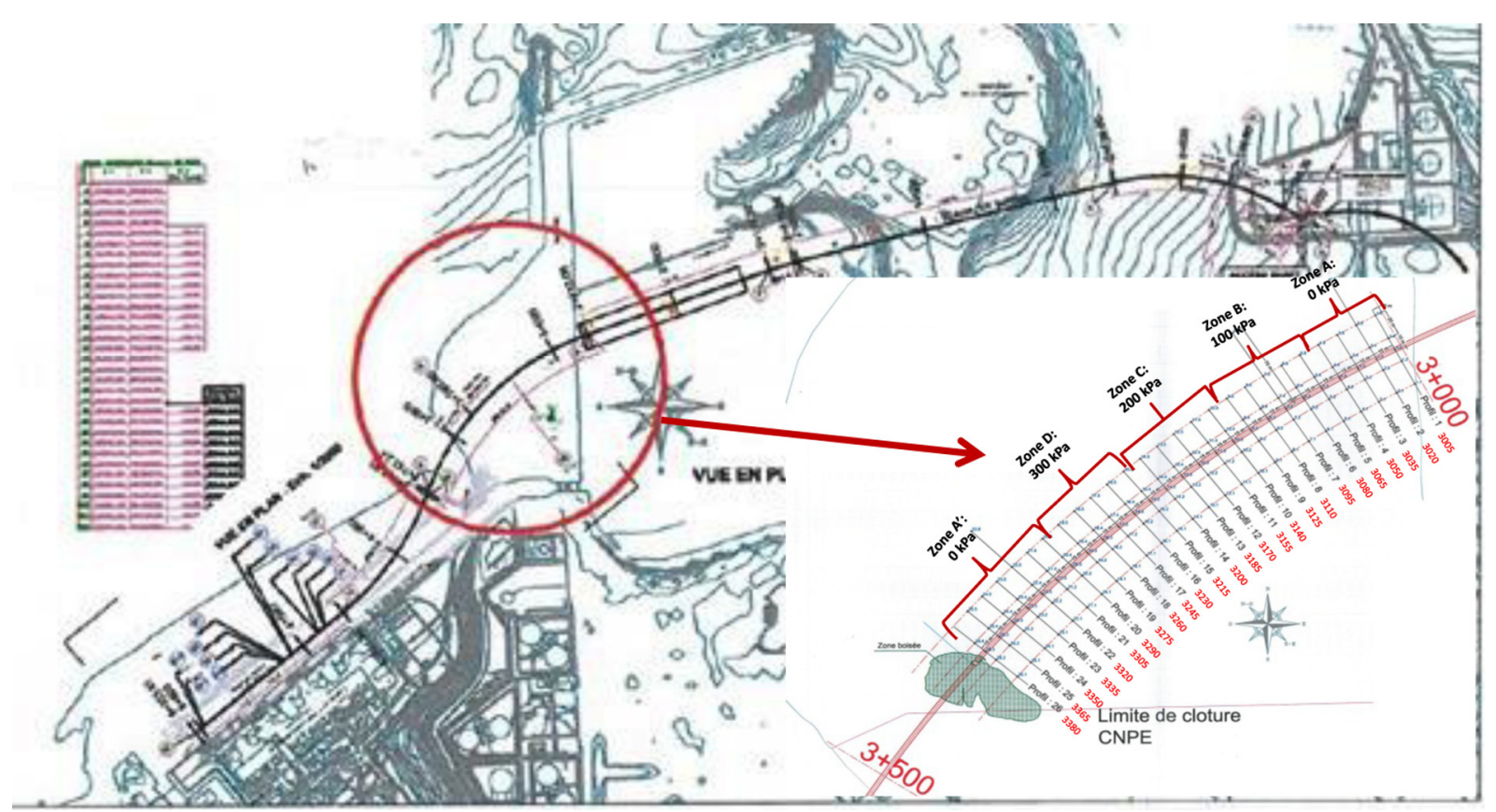

Fig. 15. Vue en plan du projet avec localisation de la zone d'auscultation avec les différents tronçons avec variation de la pression de confinement.

Fig. 15. Top view of the project with the monitoring section localization with the different confinement pressure zones.

selon les principes déjà énoncés par Arsitaghes et Autuori (2001). Ceci permet, au moins, de prendre en compte le gradient vertical de la pression et d'avoir un déconfinement variable autour de l'excavation.

\section{Excavation au «tunnelier pressurisé »: galerie hydraulique - centrale nucléaire de Gravelines}

Comme déjà énoncé dans le chapitre précédent, le creusement au tunnelier pressurisé constitue une problématique complexe à étudier. Les déplacements générés par l'excavation sont influencés, en effet, par différents facteurs, qui doivent être pris en compte, en phase d'étude, pour chercher à simuler correctement le processus d'avancement du tunnelier. Comme déjà montré par différents auteurs (Möller, 2006; Dias et Kastner, 2012; Mollon et al., 2013; Do et al., 2013), l'approche tridimensionnelle est celle qui permet de se rapprocher le plus de la complexité de ce problème.

On présente, dans ce chapitre, l'étude réalisée récemment avec Cofiva (EDF), en phase de travaux, concernant la construction d'un tunnel pour transférer l'eau chaude, rejetée par la centrale nucléaire de Gravelines, jusqu'au nouveau terminal méthanier de Dunkerque (Janin et al., 2016). L'un des principaux défis du projet a été de respecter le seuil de tassement maximal de $10 \mathrm{~mm}$, imposé au niveau de la centrale nucléaire. Afin de vérifier les études réalisées par le groupement titulaire des travaux (Bessac - Razel - Soletanche Bachy) et de s'assurer que le seuil de tassement ne soit pas atteint, une zone d'auscultation a été mise en place dans une langue de terre, avant l'arrivée du tunnelier au niveau de la centrale (Fig. 15). Des simulations numériques ont été également réalisées selon la «méthode 3D simplifiée », présentée dans le chapitre précédent. Dans la zone de

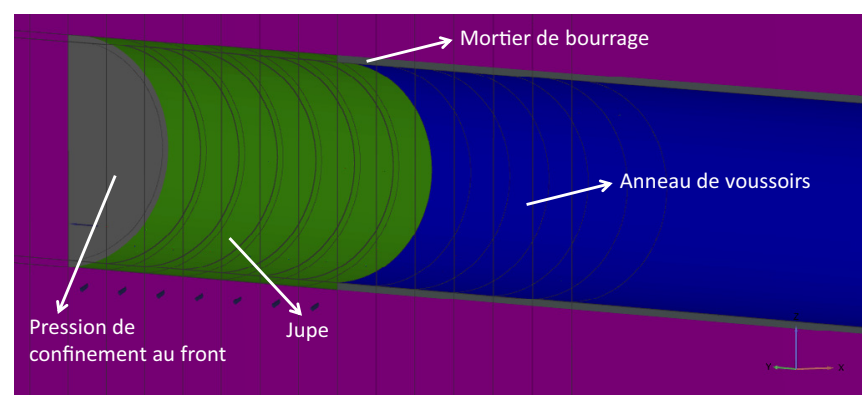

Fig. 16. Simulation 3D du processus d'avancement du tunnelier galerie hydraulique, centrale nucléaire de Gravelines.

Fig. 16. $3 D$ simulation of EPB-TBM progress - hydraulic tunnel, Gravelines nuclear power plant.

creusement sous le triangle de terre, un système d'auscultation de mesure des tassements en surface a été mis en place. Il est composé de cinq points par profils avec $15 \mathrm{~m}$ d'inter-distance entre deux profils consécutifs. La zone a été subdivisée en cinq sous-tronçons où on a fait varier la pression de confinement de consigne du tunnelier, à pression de terre, entre 0 et $300 \mathrm{kPa}$.

Le tunnel, ayant un diamètre de $3,7 \mathrm{~m}$, a été excavé dans les argiles de Flandres $\left(E_{\mathrm{CT}}=46 \mathrm{MPa}, C u=140 \mathrm{kPa}\right.$ et $\phi_{u}=0^{\circ}$, $\left.K_{0}=1,1\right)$ sous une couverture de $43 \mathrm{~m}$, composée principalement de dépôts du Quaternaire.

Avant l'arrivée du tunnelier sous la zone d'auscultation, des simulations numériques 3D ont été réalisées pour évaluer l'impact de la pression de confinement sur les tassements générés en surface. Dans la modélisation, les facteurs suivants ont été pris en compte de façon explicite (Fig. 16):

- pression de confinement au front, en la faisant varier entre 0 et $300 \mathrm{kPa}$; 
A

\section{Profil de tassement longitudinal}

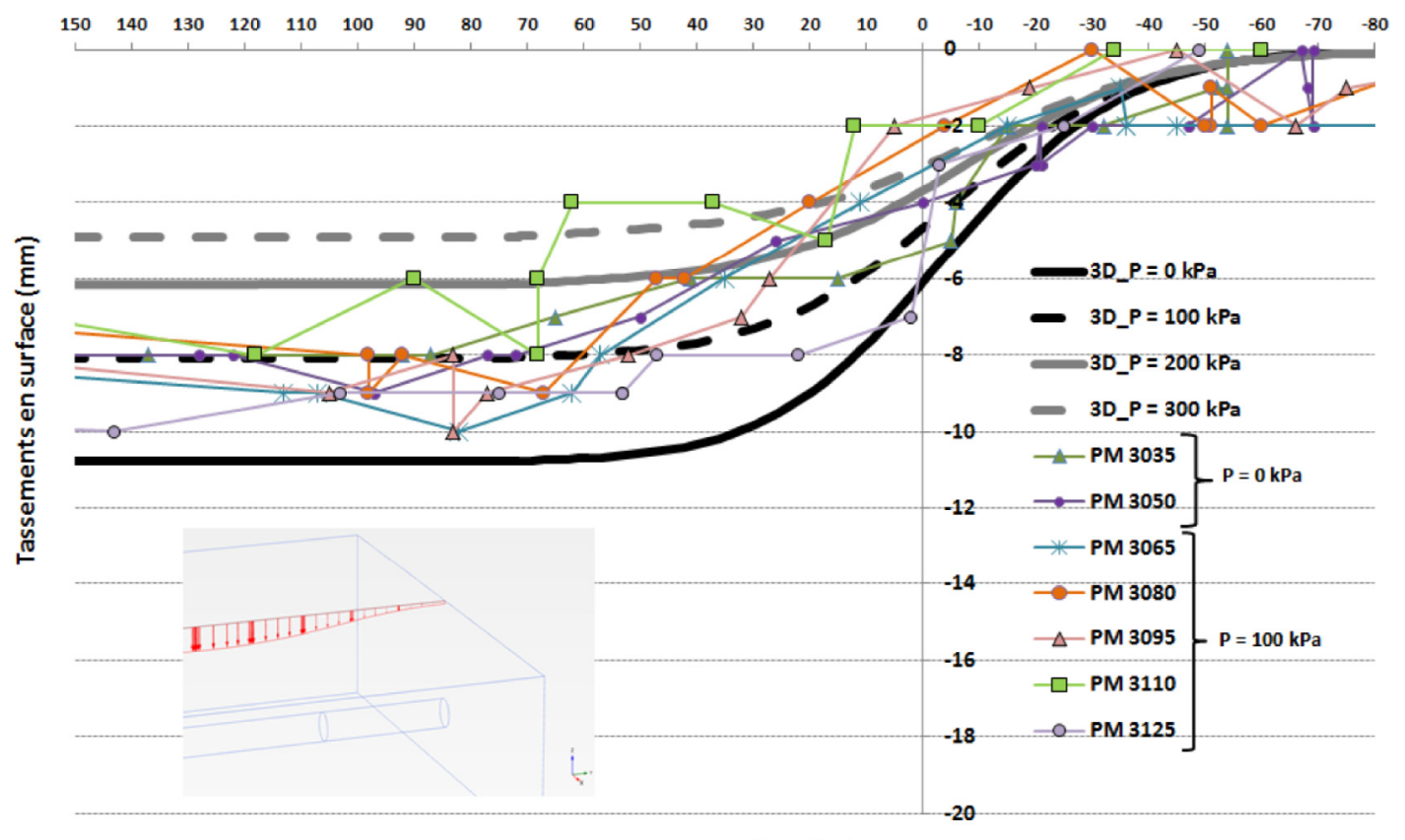

Distance au front ( $\mathrm{m}$ )

B

Profil de tassement longitudinal

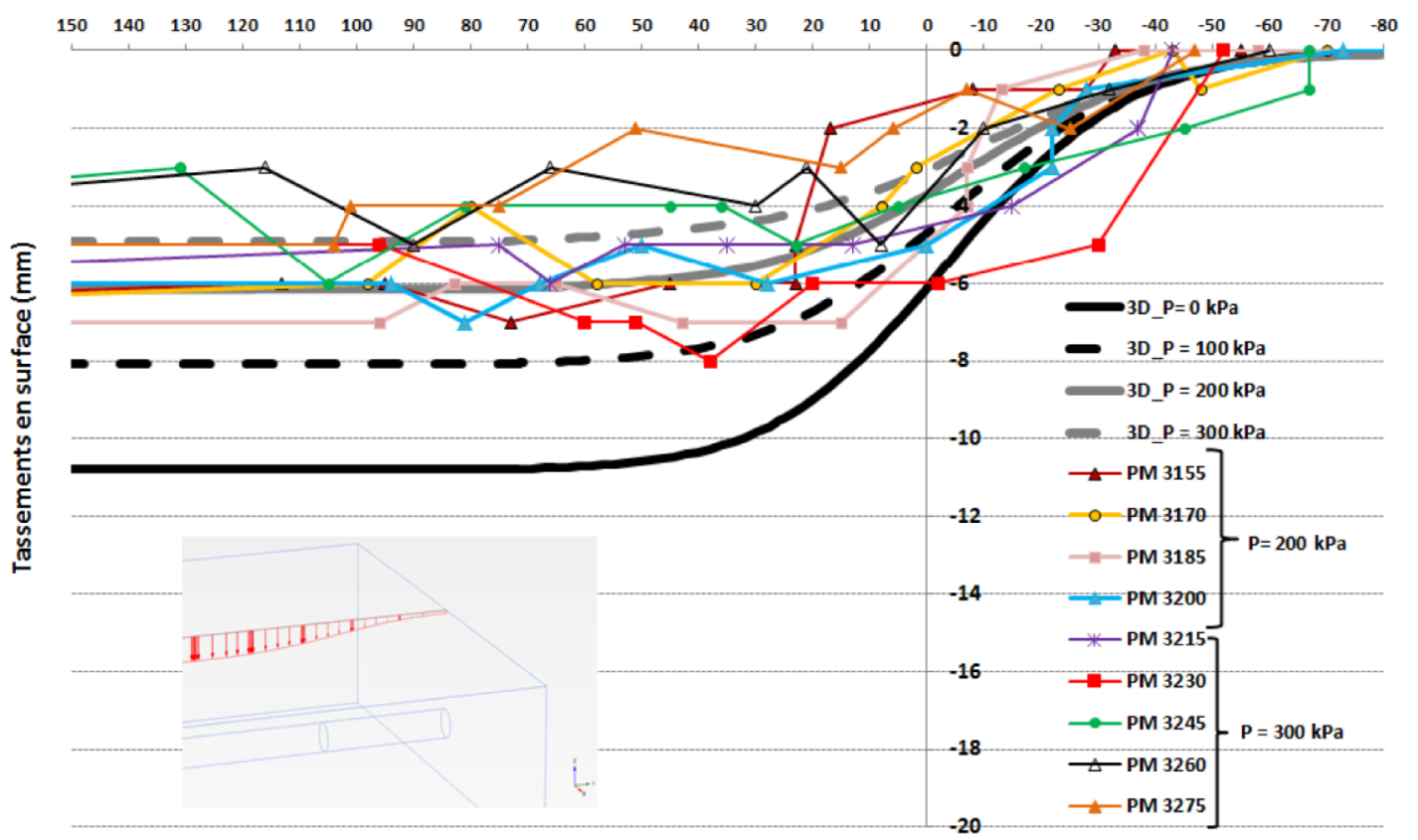

Distance au front ( $\mathrm{m})$

Fig. 17. Profil en long des tassements avec une pression de confinement de 0 et $100 \mathrm{kPa}$ (A) et de 200 et $300 \mathrm{kPa}(\mathrm{B})$ - comparaison entre les mesures in situ et les courbes prévisionnelles numériques.

Fig. 17. Longitudinal surface settlement evolution with a confinement pressure of 0 and $100 \mathrm{kPa}(\mathrm{A})$ and 200 and $300 \mathrm{kPa}(\mathrm{B})-\mathrm{comparison}$ between in situ measurements and numerical predictions. 
- présence de la jupe et de sa conicité (longueur de $8 \mathrm{~m}$ et conicité de $25 \mathrm{~mm}$ sur le rayon);

- mortier de bourrage entre le massif et l'extrados des voussoirs (modélisé de façon simplifié par des éléments volumiques);

- voussoirs (plaque élastique, $E=34 \mathrm{GPa}$ et épaisseur de $20 \mathrm{~cm})$.

En particulier, il a été essentiel de modéliser la conicité de la jupe (approche en déformations imposées). Les premiers calculs, simulant une pression de confinement également autour du bouclier (approche en contraintes), ont montré en effet que, même avec une pression de $300 \mathrm{kPa}$, le sol venait potentiellement en contact avec la jupe et il convergeait plus de l'espace annulaire réellement disponible. Ce phénomène a été confirmé par des observations in situ: à travers une trappe on voyait, en effet, l'argile venir en contact du bouclier.

Selon les principes de la «méthode 3D simplifiée», présentée dans le chapitre précédent, l'évolution des tassements en surface a pu être obtenue pour les différentes pressions de confinement: le tassement maximal varie dans ce cas entre $5 \mathrm{~mm}$ et $11 \mathrm{~mm}$ pour des pressions de confinement allant de 0 à $300 \mathrm{kPa}$. Celle-ci a été ensuite comparée aux mesures enregistrées in situ dans la zone d'auscultation. Il en résulte que la simulation numérique adoptée a été capable de très bien représenter la réponse du massif avec une très bonne correspondance, entre les courbes numériques prévisionnelles de tassement et les mesures in situ (Fig. 17). Grâce à ces résultats, il a été possible de déterminer la pression optimale de confinement du tunnelier, qui a permis ensuite de respecter le critère de tassement imposé pour la centrale nucléaire.

Cette étude a permis d'avoir une validation sur terrain de la «méthode numérique 3D simplifiée». L'importance de chercher à simuler au mieux les différents facteurs de l'excavation mécanisée au tunnelier est mise en évidence. En particulier, dans ce cas, la modélisation de la présence de la jupe et de sa conicité a été un des éléments clés de la réussite de l'étude. Dans des terrains ayant des faibles caractéristiques géotechniques, la jupe joue en effet un rôle fondamental, en venant bloquer les déplacements radiaux et ainsi le déconfinement du massif. Malheureusement, dans les calculs numériques, en particulier $2 \mathrm{D}$, ce phénomène est souvent oublié, ce qui amène à un déconfinement trop élevé et ainsi à une sur-estimation des déplacements dans le massif et à une sous-estimation des efforts dans le revêtement (Demay, 2016).

\section{Conclusions}

L'évolution des calculateurs et des codes de calcul permet aujourd'hui une «démocratisation» des approches 3D depuis le domaine universitaire vers le domaine des bureaux d'étude. Cet article présente ainsi certaines problématiques, concernant les ouvrages souterrains, dans lesquels la modélisation 3D représente un outil désormais indispensable. Elle permet, bien sûr, d'étudier des ouvrages géométriquement complexes et l'interaction avec les ouvrages existants en surface et en souterrain. Elle simule plus correctement les évolutions des contraintes autour du front de taille, les déplacements dans le massif et le chargement du soutènement et du revêtement. En outre, dans le domaine des tunnels réalisés en méthode conventionnelle, la simulation 3D est capable de mieux prendre en compte le phasage réel des travaux, la mise en place des présoutènements, une excavation divisée et multi-phasée. De même, les différents facteurs qui rentrent en jeu lors de l'excavation mécanisée au tunnelier peuvent être pris en compte de façon beaucoup plus rigoureuse que les approches bidimensionnelles. À ce propos, une «méthode 3D simplifiée » est présentée. Celle-ci a été testée, en phase d'étude d'exécution, pour estimer les tassements générés en surface et la comparaison avec les mesures enregistrées in situ a été très satisfaisante.

Les différents exemples présentés montrent également comment la simulation numérique 3D permet de mieux définir les principes du système d'auscultation à mettre en place lors de travaux: type de mesures et instruments les plus adaptés, positionnement des auscultations et définition des seuils. Les observations in situ, lors des travaux, seront ensuite essentielles pour pouvoir vérifier la pertinence des différentes hypothèses prises en compte dans le modèle numérique. Il faut, en effet, garder à l'esprit que même la simulation la plus complexe imaginable restera toujours une simplification du phénomène réel. Dans les différentes étapes du projet, l'observation (caractérisation du massif et suivi du comportement de l'ouvrage) et la modélisation numérique doivent ainsi être toujours couplées afin de bien mener à terme le projet (Guilloux, 2016). La mise en place, lors des travaux, de la méthode observationnelle est donc essentielle pour pouvoir vérifier et recaler les modèles réalisés et, en même temps, nous permettre d'améliorer les approches de simulation à adopter pour de futurs projets.

\section{Références}

Arsitaghes P, Autuori P. 2001. Calculs des tunnels au tunnelier. Rev Fr Geotech 97: 31-40.

Attewell PB. 1977. Ground movements caused by tunnelling in soil. In: Conference on Large Ground Movements and Structures, Cardiff (United Kingdom), pp. 812-984.

Clough W, Schmidt B. 1981. Design and performance of excavations and tunnels in soft clay. In: Soft Clay Engineering. Amsterdam: Elsevier Scientific, pp. 569-634.

Clough GW, Shirasuna T, Finno RJ. 1985. Finite element analysis of advanced shield tunneling. In: 5th Int Conf Numer Methods Geomech, Nagoya (Japan), pp. 1167-1174.

Dano C. 2014. Étude du creusement de tunnels au tunnelier dans les Argiles Plastiques de la région parisienne. Thèse professionnelle MASTER AFTES.

Demay B. 2016. Calcul des tunnels au tunnelier sans taux de déconfinement : est-ce possible, voire souhaitable ? In : Journées Nationales de Géotechnique et de Géologie de l'Ingénieur, Nancy (France).

Dias D. 2011. Convergence-confinement approach for designing tunnel face reinforcement by horizontal bolting. Tunn Undergr Sp Tech 26: 517-523.

Dias D, Kastner R. 2012. Movements caused by the excavation of tunnels using face pressurized shields - Analysis of monitoring and numerical modeling results. Eng Geol 152: 17-25.

Do NA, Dias D, Oreste P, Djeran-Maigre I. 2013. Three-dimensional numerical simulation for mechanized tunnelling in soft ground: the influence of the joint pattern. Acta Geotech 9: 673-694.

Eclaircy-Caudron S, Dias D, Chantron L, Kastner R. 2006. Numerical modeling of a reinforcement process by umbrella arch. In: International Conference on Numerical Simulation of Construction 
Processes in Geotechnical Engineering for Urban Environment (NSC06), Bochum (Germany).

Guilloux A. 2016. Les projets d'ouvrages géotechniques : apports de l'observation et de la modélisation. Rev Fr Geotech 146: 1-9.

Hejazi Y, Dias D, Kastner R. 2008. Impact of constitutive models on the numerical analysis of underground constructions. Acta Geotech 3: $251-258$.

Janin JP. 2012. Tunnels en milieu urbain : prévisions des tassements avec prise en compte des effets de pré-soutènements (renforcement $\mathrm{du}$ front de taille et voûte-parapluie). Thèse Doctorat, INSA de Lyon (France).

Janin JP, Dias D, Kastner R, Emeriault F, Le Bissonnais H, Guilloux A. 2011. Settlement monitoring and tunneling process adaptation case of South Toulon Tunnel. In: International Symposium on Geotechnical Aspects of Underground Construction in Soft Ground, Rome (Italy).

Janin JP, Martin A, Gastebled O. 2014. 2D and 3D numerical analysis for the design of a TBM dismantling cavern to be excavated under sensitive buildings for Paris metro line extension. In: NUMGE 2014, Delft (Netherlands).

Janin JP, Dias D, Emeriault F, Kastner R, Le Bissonnais H, Guilloux A. 2015. Numerical back-analysis of the southern Toulon tunnel measurements: a comparison of 3D and 2D approaches. Eng Geol 195: 42-52.

Janin JP, Renier P, Bergère A, Le Bissonnais H. 2016. Influence of EPB-TBM heading confinement pressure on surface settlements: comparison between 3D FE numerical predictions and in situ measurements. In: The World Tunnel Congress WTC, San Francisco (USA).

Kastner R, Ollier C, Guibert G. 1996. In situ monitoring of the Lyons metro D line extension. Geotechnical Aspects of Underground construction in Soft Ground. London: Balkema, 701-716
Lunardi P. 2008. Design and construction of tunnels - Analysis of controlled deformation in rocks and soils (ADECO-RS). Berlin (Germany): Springer.

Mair RJ, Taylor RN. 1997. Bored tunnelling in the urban environment. In: Proceeding of the 14th International Conference on Soil Mechanics and Foundation Engineering. Rotterdam: Balkema.

Möller SC. 2006. Tunnel induced settlements and structural forces in lining. PhD Thesis, Univ Stuttgart, Inst f Geotechnik (Germany).

Möller SC, Vermeer PA. 2008. On numerical simulation of tunnel installation. Tunn Undergr Spa Tech 23: 461-475.

Mollon G, Dias D, Soubra A. 2013. Probabilistic analyses of tunneling induced ground movements. Acta Geotech 8: 181-199.

O'Reilly MP, New BM. 1982. Settlements above tunnels in the United Kingdom - their magnitudes and prediction. In: Tunnelling '82. London (England): IMM, pp. 173-181.

Panet M. 1995. Le calcul des tunnels par la méthode convergenceconfinement. Paris: Presse de l'École nationale des Ponts et Chaussées.

Peck RB. 1969. Deep excavations and tunnelling in soft ground. In: Proceeding of the 7th International Conference on Soil Mechanism Foundation Engineering, Mexico, Vol. 3, pp. 255-290.

Rankin WI. 1988. Ground movements resulting from urban tunneling: predictions and effects. In: Proc Conf Engineering Geol Underground Movements, Nottingham (England), pp. 79-92.

Schmidt B. 1969. Settlements and ground movements associated with tunnelling in soil. Thèse Doctorat, University of Illinois (USA).

Serratrice JF, Magnan JP. 2002. Analyse et prévision des tassements de surface pendant le creusement du tunnel nord de la traverse souterraine de Toulon. BLPC 237: 5-36.

Volkmann GM, Button EA, Schubert W. 2006. A contribution to the design of tunnels supported by a pipe roof. In: Proc 41st U.S. Rock Mechanics Symp, American Rock Mech Assoc, Golden, Colorado.

Citation de l'article : Jean-Pierre Janin. Apports de la simulation numérique tridimensionnelle dans les études de tunnels. Rev. Fr. Geotech. $2017,150,3$. 